\title{
EL AMOR EN LA TRADUCCIÓN: TRAVESURAS DE LA NIÑA MALA DE MARIO VARGAS LLOSA Y LA NOVELA EUROPEA DECIMONÓNICA
}

\author{
Love in Translation: Mario Vargas Llosa's Travesuras de la niña mala \\ and the Nineteenth Century European Novel
}

\author{
Nataly Tcherepashenets*
}

\begin{abstract}
RESUMEN
En múltiples ocasiones Mario Vargas Llosa expresó su fascinación por las novelas del siglo diecinueve. En este trabajo voy a demostrar que Travesuras de la niña mala (2006) entra en el diálogo lúdico implícito con Madame Bovary (1856) y L'éducation sentimentale (1869) de Gustave Flaubert, y con Padres e Hijos (1862) de Ivan Turgenev, unas de las lecturas predilectas de Vargas Llosa desde su juventud. Propongo que mientras que a Flaubert y Turgenev les interesaban primeramente los aspectos psicológicos de los personajes y la exploración del poder omnipotente de los sentimientos, en la obra de Vargas Llosa, estos temas, obtienen un significado metaficticio explícito, lo que permite al escritor peruano celebrar la ficción como una fuente de la inspiración incesante, la prenda de su inmortalidad. Además, sugiero que dichos escritores comparten el escepticismo sobre la política como una fuerza renovadora de la sociedad, lo que en el caso de Travesuras de la niña mala puede ser interpretado como una manifestación artística del pesimismo del escritor frente a cualquier actividad política- una visión que marca su literatura más reciente.
\end{abstract}

Palabras clave: la novela psicológica decimonónica, metaficción, Perú, Gustave Flaubert, Ivan Turgenev.

\begin{abstract}
On multiple occasions, Mario Vargas Llosa expressed his fascination with $19^{\text {th }}$ century novels. In this article, I suggest that Travesuras de la niña mala (2006) enters into implicit dialogue with Madame Bovary (1856) and L'éducation sentimentale (1869) by Gustave Flaubert, and with Fathers and Sons (1862) by Ivan Turgenev, which are some of favored works fromVargas Llosa's youth. Whereas Flaubert and Turgenev were primarily interested in the psychological aspects of their characters and in the exploration of the omnipotent power of sentiments, in Vargas Llosa's novel these themes obtain a metapoetical significance that enables the Peruvian writer to celebrate reading as an incessant source of inspiration. I also suggest that these writers share a skepticism of politics as a reinvigorating social force, which, in the case of Vargas Llosa can be interpreted as an artistic manifestation of the writer's pessimism towards any political activity, a view that informs his latest work.
\end{abstract}

Key Words: nineteenth century psychological novel, metafiction, Peru, Gustave Flaubert, Ivan Turgenev.

State University of New York, Empire State College. Associate Professor and Area Coordinator. USA.

Correo electrónico: Nataly.Tcherepashenets@esc.edu

Recepción: 04/08/15 Aceptación: 22/10/15. 
Next to the right to create, the right to criticize is the richest gift that the liberty of thought and speech can offer.

\section{Lectures on Russian Literature} Vladimir Nabokov

... [W] hat I have read is far more important than what I have written

"A Poet's Creed," This Craft of Verse Jorge Luis Borges

En su discurso Nobel (2010), "Elogio de la lectura y la ficción," Mario Vargas Llosa confiesa que aprender a leer ha sido "la cosa más importante" que ha pasado con él en su vida $^{1}$. En múltiples ocasiones Mario Vargas Llosa expresó su fascinación por las novelas del siglo diecinueve. En su entrevista con Deborah Solomon para el New York Times en 2007, confesó que cualquier afinidad o paralelismo, que uno pueda encontrar entre su obra y la novela decimonónica, le haría sentir satisfecho y halagado. En este trabajo voy a demostrar que Travesuras de la niña mala (2006) entra en el diálogo lúdico implícito con Madame Bovary (1856) y L' éducation sentimentale (1869) de Gustave Flaubert, y con Padres e Hijos (1862) de Ivan Turgenev, unas de las lecturas predilectas de Vargas Llosa desde su juventud. Propongo que mientras que a Flaubert y Turgenev les interesaban primeramente los aspectos psicológicos de los personajes y la exploración del poder omnipotente de los sentimientos, en la obra de Vargas Llosa, estos temas, obtienen un significado metaficticio explícito, lo que permite al escritor peruano celebrar la ficción como una fuente de la inspiración incessante, la prenda de su inmortalidad ${ }^{2}$. Además, sugiero que dichos escritores comparten el escepticismo sobre la política como una fuerza renovadora de la sociedad, lo que en el caso de Travesuras de la niña mala puede ser interpretado como una manifestación artística del pesimismo del escritor frente a cualquier actividad política. Una visión que marca su literatura más reciente ${ }^{3}$.

En el prefacio a su trabajo programático, La orgia perpetua: Flaubert y Madame Bovary, Vargas Llosa explica que, este libro, es tanto sobre Flaubert y Emma, como sobre él mismo. Después de leer Madame Bovary, en 1959, supo exactamente qué tipo del escritor sería. Emma se transforma para Vargas Llosa como para León Dupuis en "l'amoureuse de tous les romans, l'héroïne de tous les drames, le vague elle de tous les volumes de vers" (La orgia perpetua $1975: 18^{4}$ ).

Leyendo Travesuras de la niña mala, uno puede observar que, en contraste con Rodolphe y León, los amantes veleidosos de Emma, Vargas Llosa permanence fiel a Madame Bovary después de casi cinco décadas. Como dice en la conversación con Solomon, le encanta la niña mala que comparte con madame Bovary el carácter non-conformista, el desprecio por las normas sociales hipocríticas y el apetito inagotable por la riqueza.

Aunque la rebeldía de Emma y Otilia tienen raices comunes: ninguna de las dos mujeres quiere resignarse a su suerte; hay una distancia que separa estos personajes. Mientras que Emma, el personaje inspirado en las novelas románticas, busca una vida amorosa excitante y confunde el amor con el sexo, las aspiraciones de Otilia son mucho más terrestres y pragmáticas: quiere romper con su origen de "una chica muy humilde" (37) para siempre. Otilia, cuyo nombre, de Odelia significa en hebreo 'alaba a dios' -lo que implica subordinación y humildadirónicamente, características totalmente ajenas al personaje creado por Vargas Llosa, según su padre, "tenía delirios de grandeza desde que nació [...] no se conformaba con su suerte" y "siempre soñó con lo que no tenía" (2006: 319).

Otilia, al igual que Emma, niega la posibilidad del amor en la pobreza y desprecia la estabilidad de la vida mediocre. Como Vargas Llosa observa, Emma es una encarnación del mundo moderno, donde las personas muchas veces se transforman en los esclavos de objetos. En Madame Bovary, "Amor y dinero se apoyan y activan mutuamente" (1975: 161). De una manera parecida, la niña mala, asocia la libertad con la fortuna y según el narrador, "[está] dispuesta a todo para no ser vencida y seguir escalando posiciones" (2006:1315). Además, las dos tienen una atracción por la transgresión y 
ejemplifican una idea de que para afirmarse en la vida uno tiene que arriesgarse. Una tiene que imaginarse o exponerse al riesgo. En el caso de Vargas Llosa, este motif puede ser interpretado además como una manifestación de la observación de Kristal, que sugiere que, el escritor peruano sigue a Georges Bataille en su idea de que, sin una transgresión, la realización del placer es imposible 6 .

El rechazo de las normas sociales es parte de esta transgresión, la que en la imaginación de ambos personajes les va a permitir escapar de la realidad mediocre. Además, las dos piensan que cualquier método es válido para alcanzar su meta: "Para conseguir lo que se quiere, todo vale" (2006: 33). El efecto dramático, sin embargo, se crea por que hay un intervalo entre la ilusión y la realidad, la distancia entre el deseo y su cumplimiento. En el caso de Emma, su ideal amoroso, creado a través de sus lecturas, es destruido por una vida de adulterio y el aburrimiento de su matrimonio. En el caso de la niña mala, su ambición de romper con su pasado, de saltar a otra clase social, le cuesta su salud mental y fisica. Evocando la visión de Foucault (1986), según la cual la sexualidad está saturada en el poder, y que el pago por el último puede traer daño a la salud del individuo, según el narrador, "[s]ólo lo había conseguido por cortos períodos y lo había pagado carísimo, dejando pedazos de su piel y de su alma en el camino" (2006: 323-324).

Además, en la novela de Vargas Llosa este efecto dramático está enfatizado y subvertido a la vez, por el aspecto metaficticio de la novela y la función dismistificadora de la ironía. Así, la niña mala, casi explícitamente, trata de re-inventarse a sí misma através de sus multiples casamientos sin amor y nombres robados de la ficción ${ }^{7}$. Uno de éstos es Madame Robert Arnoux, lo que inmediatamente nos hace recordar el amor inalcanzable de Frederic, el protagonista de $L$ ' éducation sentimentale de Flaubert. Ricardo Somocurio tiene la misma asociación, la cual provoca un efecto irónico: "En los huecos libres releía L' éducation sentimentale, de Flaubert, porque, ahora, la madame Arnoux de la novela tenía para mí no solo el nombre, también la cara de la niña mala" (2006: 64). La niña mala, además, parece ser casi consciente de su precursora literaria, y juega con el paralelismo, cuando se presenta ante Ricardo como una esposa modelo de la reputación impecable y se comporta así durante los primeros cinco minutos. Mientras que Mme Arnoux de Flaubert es una encarnación de la virtud, los valores del matrimonio y los hijos; la niña mala, el amor de la vida de Ricardo, Mme Arnoux, creada por Vargas Llosa, es todo lo contrario. Siempre está a la búsqueda de aventuras y la estabilidad de la vida matrimonial la deprime. El paralelismo ejemplifica una parodia que, según Linda Hutcheon, es una repetición con distancia irónica.

El diálogo lúdico con su "dios particular," lo que es Flaubert para Vargas Llosa, como José Miguel Oviedo señala, continúa más allá de las afinidades entre los personajes. Como Vargas Llosa nota, aunque Flaubert evitaba todo lo accidental y dramático, y tenía orgullo en crear la literatura apoyada en "la normalidad," los elementos románticos tenían cierta atracción para él: "Quasimodo también cruza fugazmente las calles de Yonville convertido en un ciego de pústulas y Emma debe algunos de sus rasgos a la preciosa gitanilla romántica"(1975: 249). Vargas Llosa sugiere que una incorporación de los elementos mágicos o poco explicados, engrandecen el realismo y le dan "un nuevo impulso al designio totalizador del género novelesco"(1975: 249).

Los elementos semejantes a cierto indicativo mágico aparecen en el cuadro realista de la niña mala, para enfatizar su carácter extraordinario. Es la hija de Arquímedes, quien irónicamente tiene el nombre de uno de los más notables científicos y matemáticos de la antigüedad, y al contrario del principio discubierto por Arquímedes de Grecia, no puede 'flotar' y vive en la pobreza extrema. Sin embargo, tiene la habilidad única de adivinar las intenciones del mar y aconseja a los ingenieros donde es mejor construir el rompeolas. Otilia parece heredar algunas de las facultades mágicas de su padre, y le devuelve la voz a un niño mudo vietnamita. Propongo que, como en el caso de 
Flaubert, estas habilidades extraordinarias de índole romántico no contradicen al realismo, un género privilegiado para Vargas Llosa, sino que lo enriquecen.

Los dos escritores comparten la idea de que el novelista inventa historias a partir de su historia personal. Una de éstas, inspiradoras de Travesuras de la niña mala, sugiero, es la pasión de su autor por la lectura, la que provee el impulso creativo y sigue enriqueciendo tanto su realidad como su ficción ${ }^{8}$. No es por casualidad que el narrador en primera persona, es un lector ávido. Ricardo Somocurio es también el traductor y el intérprete, quien tiene un interés especial por la literatura rusa, la cual quiere introducir en el mundo hispanohablante: "Mi amigo Muchnik había tratado de conseguir alguna traducción del ruso -era lo que más me ilusionaba-, estuvimos a punto de convencer a un editor a que se animara a publicar Padres $e$ hijos de Turgenev" (2006: 334).

El escritor ruso, Ivan Turgenev, fue un amigo íntimo de Flaubert durante más de veinte años, y sus cartas abren el volumen de la correspondencia del escritor francés, que Vargas Llosa compró cuando tenía veinticuatro años, como recuerda en La orgía perpetua ( $\mathrm{p}$. 14). En aquel tiempo también empezó a leer las obras de Turgenev, y se puede ver que, en Travesuras de la niña mala, entra en un diálogo lúdico con su novela más famosa Padres e hijos. Así, la niña mala y Bazaroff, el protagonista de Padres e hijos, comparten el nihilismo, el rechazo de cualquier autoridad y la negación del poder de las emociones. Bazaroff es un médico, quien considera los matrimonios por dinero como una cosa muy razonable y piensa que los sentimientos son una enfermedad, y también desprecia las convenciones sociales y las ilusiones románticas. De una manera parecida, la niña mala, parece rechazar todo lo romántico y racionalizar unas relaciones más íntimas. Según el narrador, "Hablaba con tanta frialdad que no parece una muchacha haciendo el amor, sino un médico que formula una descripción técnica y ajena al placer" (Travesuras 67). Como Bazarov, la niña mala desprecia todo tipo de enlaces emocionales y, parece que la única constante que tiene en su vida, es su relación fragmentaria y caótica con Ricardo ${ }^{10}$.

Por otra parte, la niña mala, así como Bazaroff, tienen una necesidad escondida por lo sentimental. Bazaroff se enamora de Odinzova y a la niña mala le encanta escuchar huachaferías ${ }^{11}$. Tanto la muerte de Bazaroff como la enfermedad incurable de Otilia pueden ser interepretadas, a nivel simbólico, como una destrucción causada por el rechazo de las emociones, por el intento de subordinar la vida a metas puramente pragmáticas. Tanto Vargas Llosa como Turgenev reafirman el poder inevitable de los sentimientos. Mientras que Turgenev explora este tema como un aspecto del realismo psicológico, el escritor peruano lo introduce explícitamente como un tópico literario eterno. Así como la niña mala sugiere, va a continuar viviendo e inspirar a Ricardo después de su muerte como una protagonista de su novela todavía no escrita: "Por lo menos, confiesa que te he dado tema para una novela. ¿No, niño bueno?" (Travesuras 375). Así nunca va a quedarse sólo; el círculo lectura-vida- creación-vidalectura obtiene una importancia ontológica.

$\mathrm{Al}$ contraste con la literatura, la política ${ }^{12}$ fracasa en aportar renovación a la vida de un individuo. En su carta a la condesa Lambert, Turgenev reafirma su aversión por la politica. En su carta a George Sand, Flaubert observa que la era de la politica se acabó y que todos los partidos y regímenes se parecen en que van más atrás de su época ${ }^{13}$. En su libro, que precede a Travesuras de la niña mala, La tentación de lo imposible: Victor Hugo y Los Miserables (2004), Vargas Llosa se refiere a $L^{\prime}$ éducation sentimentale como una obra cargada de pesimismo social y político. Lo mismo se puede decir sobre la novela del escritor peruano. La futilidad de la posición politica activa es uno de los tópicos centrales en $L$ 'éducation sentimentale y Travesuras de la niña mala. En la novela de Flaubert, las esperanzas y el entusiasmo durante el período de diciembre de 1841, asociados con la posibilidad de establecer la democracia y terminar el reinato del rey Louis 
Phillipe, se cambian por las turbulencias de 1848. La creación de la segunda Republica, llevó a la tensión entre la République démocratique et sociale la que resultó en la represión y el desafío. Surge la desilusión que marca el final de la vida de Frederic.

Ricardo, al igual que Frederic, aprende que la política es un ejercicio de futilidad. En Travesuras de la niña mala, los discursos revolucionarios febriles se convierten en acciones vacías. Por ejemplo, "las cartas del tío Ataúlfo", que Ricardo recibe de Perú, suenan "cada día más desesperanzadas" (Travesuras 150). Las aspiraciones asociadas con la transición a la democracia fracasan. En vez de transición a la democracia después de la dictadura de Francisco Morales Bermudez, "La sociedad peruana se iba sumiendo cada vez más en la pobreza, la ignorancia y la brutalidad" (Travesuras 151). Así, la novela de Vargas Llosa puede ser considerada otra expresión del escepticismo del escritor sobre la política como una fuerza que pueda mejorar la vida del individuo, el tema que parece estar presente en la obra de Vargas Llosa por lo menos desde su novela La fiesta del Chivo ${ }^{14}$.

Por eso la vida de Ricardo, así como la de su precursor literario Frederic, está marcada por la inercia y la pasividad. Una transición ilusoria, repetitiva y con falta de renovación es un letmotif en ambas novelas. Así al principio de L' éducation sentimentale, Frederic escoge una ruta más larga a su ciudad natal, en un acto simbólico que crea un retraso y un elemento de suspenso artificial. En Travesuras de la niña mala, Ricardo recorre espacios urbanos tan distintos como París, Londres, Tokyo, Madrid; lo que, por un lado, permite a Vargas Llosa, según Nataly Villena Vega, prefigurar literariamente "un cosmopolitismo temático a partir de la inmigración" y según Joaquín Marcos, crear una novela en un "espacio global," a diferencia de todas las novelas anteriores del escritor peruano, pero por otro lado, los viajes no traen casi ningún cambio en la vida de Ricardo, y sólo parecen reafirmar sus dos únicas ambiciones que son: la niña mala y París. Ricardo ama a Otilia, Lily la chilenita, la camarada Arlette, madame Robert Arnoux, Mrs. Richardson, Kuriko y madame Ricardo Somocurcio, que sigue siendo la niña mala, aunque cambie tantos nombres y esposos ${ }^{15}$. Trata de liberarse de ella pero sabe muy bien que no puede ${ }^{16}$. La llama "una enfermedad incurable," "una pesadilla" a la que odia y a la que adora a la vez. Sugiero que, en el nivel simbólico, la relación entre la niña mala y Ricardo puede ser interpretada como una metáfora de la relación entre Vargas Llosa y Perú. Curiosamente, en su ensayo, "El país de las mil caras," el escritor describe su relación con su país en términos semejantes: "El Perú es para mí una especie de enfermedad incurable y mi relación con él es intensa, áspera, llena de la violencia que caracteriza a la pasión" (1990: 246).

Para concluir, Travesuras de la niña mala puede ser leída como una expresión artística lograda de una relación turbulenta con su país, llena de nostalgia y disolución, pero siempre apasionada, así como una manifestación sutil, juguetona, y consciente de sí misma de toda una vida de compromiso entre el autor y la literatura decimonónica, con la que mantiene un diálogo implícito que continúa alimentando su imaginación.

\section{Notas}

1. Curiosamente, Jorge Luis Borges hizo una afirmacíon semejante en varias ocasiones, una de las más notables en "Autobiographical Essay" ("Un ensayo autobiográfico" publicado, originalmenyte en inglés, donde observa: "If I were asked to name a chief event in my life, I should say my father's library.”(1970: 209). Después de recibir el premio Nobel, el escritor peruano confesó: "Me da un poco de vergüenza recibir el premio Nobel que no recibió Borges" (2010) y dictó el curso sobre Borges en Princeton la primavera de 2011.

2. Vale la pena notar que en la entrevista que Vargas Llosa hizo a Borges en 1963, el escritor Argentino destacó a Flaubert como un escritor francés más importante para él. Borges es famoso por su desprecio por la novela psicológica expresado, por ejemplo, en su prólogo a Adolfo Bioy Casares's La invención de Morel, donde Borges observa: "La novela característica,"psicológica", proponde a ser informe"(1996:25). El juego artístico con los 
aspectos psychologicos de la novela decimonónica en la novela de Vargas Llosa puede ser interpretado como un tributo lúdico al gran escritor Argentino.

3. Según Efraín Kristal, convencimientos políticos personales de Vargas Llosa marcan su literatura. Estamos de acuerdo con su observación que despúes de que Vargas Llosa perdió en las elecciones del julio de 1990, su obra estaba expresando su escepticismo sobre la politica en general.

4. Elizabeth Sánchez Garay sugiere una lectura creativa del ensayo como "una defensa de la libertad creativa, literaria y personal” (53). Podemos añadir que esta libertad marca la ouevre de Vargas Llosa, y es un tema importante que reaparece y permite relacionar su obra creativa, su crítica literaria y su periodísmo. Así en su interpretación más reciente de Don Quijote propone que es "una canción a la libertad," uno de los rasgos que la hace una novela del siglo XXI (una novela contemporánea).

5. No por casualidad su primer nombre-máscara es "Lily" que evoca una asociación con "Lili" o la "Lilith" bíblica, la primera mujer de Adán, que, además, puede ser considerada como la primera mujer fatal en la historia de la humanidad, cuya mención aparece, en Isaiah 34:14 al lado de los animales salvajes.

Wildcats shall meet with

hyenas,

goat-demons shall call to each other;

there too Lilith shall

repose,

and find a place to rest.

Elvire Gómez -Vidal (2011) y Raisacruz Huertas Neri (2011) analizan en profundidad el significado simbólico del nombre "Lily" en la novela de Vargas Llosa.

6. En su ensayo "Bataille o el rescate del mal," Vargas Llosa afirma que comparte con Bataille sus ideas sobre la literatura como "un acto de rebeldía, un afán de recuperación de la cara oculta de la vida" (21).

7. Estamos de acuerdo con María Regina Ruiz que la niña mala ejemplifica un simulacro que según Jean Baudrillard, "no tiene un referente, un territorio o una sustancia" (145).

8. Giovanna Pollardo propone que "la niña mala," que exige todo a cambio de nada (el amor sin condiciones) es una metáfora de la presencia constante, caprichosa y hasta aditiva por la literatura en la vida del escritor. Esta percepción de la literatura es evidente en la conversación de Vargas Llosa con Cano Gaviria, donde el escritor peruano observa: "Para el escritor auténtico, escribir es una manera de vivir, algo de lo cual no puede prescindir, del mismo modo que el alcohólico no puede prescindir del alcohol ni el drogadicto de las drogas" (Cano Gaviria 25).

9. Mario Muchnik, el fundador de "Muchnik Editores," ha publicado a autores tan significativos como John Steinbeck, Leo Tolstoi, Franz Kafka, Mark Twain e Isaia Berlin.

10. Ricardo, 'el eterno enamorado' de la niña mala, evoca, sin duda, una imágen de Florentino Ariza, el protagonista de Amor de los tiempos de la cólera de Gabriel García Márquez. Esperanza Domínguez Castro analiza paralelismos temáticos y estructurales entre Travesuras de la niña mala y Memoria de mis putas tristes (2011).

11. José Miguel Oviedo asocia "huachaferías" con "lo cursi, de mal gusto, ridículo por pretencioso." Para los ejemplos más recientes de huachaferías, según Vargas Llosa, ver su entrevista con Mariella Balbi "El chisme es una pasión limeña" en El Comercio, 1 de noviembre de 2013, http://www. elcomercio.com.pe/impresa/notas/chisme-pasionlimena/20090201/239921

12. Ver, por ejemplo, la carta \#132, pp. 185-187.

13. Ver, por ejemplo, la carta \#100, pp. 92-93.

14. Nataly Tcherepashenets propone una lectura de La fiesta del Chivo en su artículo "The Secrets of the Era: the Rhetoric of Silence on Responsibility and Guilt in Mario Vargas Llosa's La fiesta del Chivo".

15. Del interés particular es su relación con el esposo japonés, Furuda, que está marcada por la crueldad extrema y resulta en la enfermedad fatal de "la niña mala," el cancer. Como sugiere Stephen Henighan, esta relación puede ser interpretada, en el nivel simbólico, como una metáfora de lo japonésperuano Alberto Fujimori hizo a la nación peruana, representada por Lily, "la niña mala.” Además, en el nivel metaliterario, Rebecca Riger Tsurumi señala que con un gangster japonés, Vargas Llosa añade a su repertoire de "unforgettable villans" (74), que también incluye a Fushía de Casa verde. 
16. El motivo del primer amor, como una experiencia cautivadora, es también recurrente en las otras obras de Turgenev, por ejemplo en "El primer amor," "Assya” y "Rudin.” Además, la presencia de la enigmática de la femme fatale es frecuente en las páginas de sus novelas; Walter Smyrniw traza el papel que cumplió el escritor ruso en la creación de la representación icónica de femme fatale, ver Walter Smyrniw's “Turgenev's Emancipated Women".

\section{Bibliografía}

Borges, Jorge Luis. (1970). "An Autobiographical Essay." En The Aleph and Other Stories, pp. 203-260. New York: E.P. Duton and Co.

. (1996). "Adolfo Bioy Casares. La Invención de Morel." Obras Completas. Vol. 4, pp. 25-27. Barcelona: Emecé Editores.

. “A Poet's Creed." (2000). This Craft of Verse. En The Charles Eliot Norton Lectures 1967-1968. Ed. Calin-Andrei Mihailescu, pp. 97-12500. Cambridge: Harvard University Press.

Domínguez Castro, Esperanza. (2011). "Vargas Llosa y Las travesuras de la niña mala, Gabriel García Márquez y Memoria de mis putas tristes: ¿epígonos de sí mismos?", Anales de Literatura Hispanoamericana, 40: 363-384.

García Márquez, Gabriel. (1996). El amor en los tiempos de cólera. Penguin Books: New York.

Flaubert, Gustave. (2000). Correspondance. Ed. Jean Bruneau. Paris: Gallimard. . (1971). Madame Bovary. Paris: Garnier. . (1968). L'éducation sentimentale. Paris: Garier.
Foucault, Michael. (1990). The History of Sexuality. Vol.1 Trans. Robert Hurley. New York: Vintage Books.

Gómez-Vidal, Elvire. (2010). "Poética de la figura femenina en la obra de Vargas Llosa." En Mario Vargas Llosa: perspectivas críticas ensayos inéditos. Eds. Pol Popovic Karic and Fidel Chávez Pérez, pp. 282-314. México: Técnológico de Monterrey.

Henighan, Stephen. (2009). "Nuevas versiones de lo femenino en La Fiesta del Chivo, El paraíso en la otra esquina y Travesuras de la Niña Mala." Hispanic Review, 77 (3): 369-388.

Huertas Neri, RaisaCruz. (2011). “¿Cuál es la travesura más fascinante de la niña mala?" Espéculo: Revista de Estudios Literarios, (47) [con acceso el 15 de julio de 2015].

Hutcheon, Linda. (1991). Narcissistic Narrative: The Metafictional Paradox. London: Routledge.

Kristal, Efraín. (1998). Temptation of the World. The Novels of Mario Vargas Llosa. Nashville:Vanderbilt University Press.

Marco, Joaquín. (2008). "Viaje literario en el mundo global: sobre Travesuras de la niña mala de Mario Vargas Llosa." En El viaje de la literatura hispanoamericana: el espíritu colombino. Eds. Sonia Mattalia, Pilar Celma y Pilar Alonso. Vervuert: Iberoamericana, 106-119.

Lowe, David (ed.). (1983). Turgenev Letters. Trans. David Lowe. Ann Arbor: Ardis Publishers.

Oviedo, José Miguel. (2009). “Travesuras de la niña mala, de Mario Vargas Llosa.” Letras libres, julio de 2006 en http://letraslibres. com/index.php?art=11369 [con acceso el 15 de marzo de 2015]. 
Pollarolo, Giovanna. (2008). "La 'niña mala' como alegoría de la vocación literaria." En Perú en el espejo de Vargas Llosa. Eds. Javier Vargas de Luna and Emilia Deffis. Puebla, Mexico: Universidad de las Américas Puebla. 71-79.

Riger Tsurumi, Rebecca. (2012). The Closed Hand. Images of the Japanese in Modern Peruvian Literature. West Lafayette: Purdue University Press.

Ruiz, María Regina. (2009). "Lo hiperreal en la novela Travesuras de la niña mala, de Mario Vargas Llosa." Hipertexto, 9: 147-152.

Sánchez Garay, Elizabeth. (2010). "La passion perpetua de Vargas Llosa por Madame Bovary."En Mario Vargas Llosa: perspectivas críticas ensayos inéditos. Eds. Pol Popovic Karic and Fidel Chávez Pérez. México: Técnológico de Monterrey. 53-81.

Tcherepashenets, Nataly. (2011). "The Secrets of the Era: the Rhetoric of Silence on Responsibility and Guilt in Mario Vargas Llosa's La fiesta del Chivo." Hispanic Journal, 32(1): 89-103.

Vargas Llosa, Mario. (1986). "Bataille o el rescate del mal." Contra viento y marea. Vol. 2, pp. 9-29. Barcelona: Editorial Seix Barral.

. (2009). "El chisme es una pasión limeña. Entrevista" El Comercio, http://www. elcomercio.com.pe/impresa/notas/chismepasion-limena/20090201/239921 [con acceso 18 de mayo, 2015].
(2010). "Elogio a la lectura y la ficción” http://www.nobelprize.org/ nobel_prizes/literature/laureates/2010/ vargas_llosa-lecture_sp.pdf [con acceso 3 de agosto, 2015].

. (1971). La historia secreta de una novela. Barcelona: Tusquets Editor.

. (1975). La orgia perpetua. Barcelona: Taurus.

. (1990). "El país de mil caras." Contra viento y marea. Vol. 3 (1964-1988), Barcelona: Seix Barral.

. (1963).“Preguntas a Borges”. En http:// www.hacer.org/pdf/Borges.pdf [con acceso 3 de agosto, 2015].

. (2007). "The Storyteller. Entrevista." The New York Times, 7 de octubre 2007 en http://www.nytimes.com/2007/10/07/ magazine/07wwln-q4-t.html?_r=1 [con acceso 10 de mayo, 2015].

. (2004). La tentación de lo imposible: Victor Hugo y Los Miserables. Madrid: Alfaguara.

(2006). Travesuras de la niña mala. Bogotá: Alfaguara.

Vega, Nataly Villena. (2007-2008). “El cosmopolitismo y su irradiación en El paraíso en la otra esquina y Travesuras de la niña mala de Vargas Llosa." Espéculo, 37 (http://www.ucm.es/info/especulo/ numero37/cosmopol.html [con acceso 3 de agosto, 2015].



Este obra está bajo una licencia de Creative Commons Reconocimiento-NoComercial-SinObraDerivada 4.0 Internacional. 